\section{BMJ Open Respiratory Research}

\title{
Safety and efficacy of ambulatory management of secondary spontaneous pneumothorax: a case series
}

\author{
Fasih Khan, Yusuf Vali, Muhammad Naeem, Raja Reddy
}

\begin{abstract}
To cite: Khan F, Vali Y, Naeem M, et al. Safety and efficacy of ambulatory management of secondary spontaneous pneumothorax: a case series. BMJ Open Resp Res 2019;6:e000373. doi:10.1136/ bmjresp-2018-000373
\end{abstract}

- Additional material is published online only. To view please visit the journal online (http://dx.doi.org/10.1136/ bmjresp-2018-000373).

Received 14 October 2018 Revised 28 January 2019 Accepted 3 February 2019

\section{Check for updates}

(C) Author(s) (or their employer(s)) 2019. Re-use permitted under CC BY-NC. No commercial re-use. See rights and permissions. Published by BMJ.

Respiratory Medicine, Kettering General Hospital NHS Foundation Trust, Kettering, UK

Correspondence to Dr Raja Reddy; rvreddy51@hotmail.com

\section{ABSTRACT}

Introduction The optimal management of pneumothorax remains undefined. There is a growing consensus that patients with spontaneous pneumothorax can be considered for ambulatory management with the use of a one-way valve. Despite this, there is little data on the outcomes of outpatient management of secondary spontaneous pneumothorax (SSP).

Methods At our institution, selected patients with primary and secondary spontaneous pneumothorax who meet the predefined local criteria are managed on an ambulatory pathway. We prospectively evaluated our practice over a 3-year period and explore outcomes of patients with SSP using primary spontaneous pneumothorax (PSP) as a comparator group.

Results 163 consecutive patients presenting to our hospital between September 2014 and July 2017 were evaluated using a predefined protocol. 111 (49 SSP and 62 PSP) were deemed suitable for outpatient management. Resolution on day 5 was similar between the two groups ( $65 \%$ in the SSP vs $79 \%$ in the PSP group; $p=0.108$ ). The mean drainage time was 5.84 days in SSP compared with 5.69 days in PSP, representing a difference of 0.15 days $(95 \% \mathrm{Cl}-2.47$ to $2.16 ; \mathrm{p}=0.897)$. Complications such as infection and drain blockage/falling-out were scarce, with comparable pain and satisfaction scores across both groups. There were no deaths during this period. An estimated £86796 (\$113 920) was saved over the study period, equating to $£ 1118.80$ (\$1550) per patient.

Discussion This study suggests that outpatient management of selected patients with SSP may be effective, safe and cost-saving.

\section{INTRODUCTION}

Spontaneous pneumothorax, defined as air in the pleural cavity in the absence of a traumatic injury to the chest, can be broadly divided into primary (PSP) and secondary (SSP) groups. SSP is associated with underlying lung disease as opposed to no underlying lung disease in PSP. It is a global health problem, with an incidence of $6-7 / 100000$ cases per annum for males and 2/100 000 for females. ${ }^{1}$ Combined hospital admission rates in the UK for both primary and secondary pneumothoraces have been reported as

\section{Key messages}

Can patients with secondary spontaneous pneumothorax be managed safely and effectively on an ambulatory pathway?

- Data on ambulatory management of secondary spontaneous pneumothorax are sparse, but suggest it could be considered in selected cases; however, the criteria for case selection are not defined.

- Ambulatory management of carefully selected patients with secondary spontaneous pneumothorax appears to have comparable outcomes with ambulatory management of primary spontaneous pneumothorax, while also appearing to be safe and cost-saving.

$16.7 / 100000$ and 5.8/100 000 in men and women, respectively. ${ }^{2}$

There remains a lack of consensus on the optimal initial management of pneumothorax, with varying recommendations between the British Thoracic Society (BTS), ${ }^{3}$ American College of Chest Physicians $(\mathrm{ACCP})^{4}$ and Belgian Society of Pulmonology. ${ }^{5}$ However, both the BTS and ACCP recognise that selected patients can be managed on ambulatory pathways with the use of Heimlich valves connected to a small chest tube.

This is not a novel concept, with small case series ${ }^{6} 7$ published over 40 years ago describing the use of Heimlich valves for the successful management of selected spontaneous pneumothoraces. In 2006, Marquette and colleagues ${ }^{8}$ again demonstrated the feasibility of using Heimlich valves in the inpatient management of patients with PSP. Subsequently Massongo et at ${ }^{9}$ extended the use of Heimlich valves to managing such patients in an ambulatory setting. Voisin $e t a l^{10}$ took this a step further and included selected SSP for ambulatory management with pigtail catheters. However, this study only comprised 22 patients. A more recent systematic review by Brims and Maskell ${ }^{11}$ suggests ambulatory management may have benefits for patient 
comfort, mobility and avoidance of hospital admission, with comparable outcomes with current practice, although the current published literature cannot reliably inform this.

At our hospital, patients (both SSP and PSP) who meet the predefined local criteria have been managed on an ambulatory pathway since 2013 with no serious incidents. The aim of the study was to explore the outcomes of managing SSP on an outpatient basis, by using patients with PSP as a comparator group.

\section{METHODS}

\section{Setting}

This study was carried out prospectively between September 2014 and July 2017 at Kettering General Hospital, Northamptonshire, in the Emergency Department and the Department of Respiratory Medicine. The institution has 640 beds and serves a surrounding population of approximately 300000 inhabitants.

All patients with spontaneous pneumothorax aged 16-80 years were considered for ambulatory management. They were classified based on the BTS criteria ${ }^{3}$ into PSP (no underlying respiratory disease) or SSP (aged $>50$ with strong smoking history or underlying respiratory disease on chest radiograph). They were also divided into small $(<2 \mathrm{~cm}$ air between the lung margin and chest wall at the level of the hilum) or large $(>2 \mathrm{~cm})$.

\section{Inclusion criteria}

All patients with PSP were deemed eligible for ambulatory management.

For patients with SSP, those who met the following criteria were deemed eligible for ambulatory management:

1. Cognitively intact.

2. WHO performance status of $0-1 .^{12}$

3. Able to attend the ambulatory care unit for follow-up.

4. No coexisting condition requiring hospital admission.

5. Aged between 16 and 80 years.

Patients with iatrogenic/traumatic pneumothorax were excluded.

\section{Patient journey}

Patients presenting to the emergency department (ED) with spontaneous pneumothorax confirmed on imaging were managed in line with the BTS guidelines. ${ }^{3}$ In PSP, manual aspiration was performed initially if the pneumothorax was large or in the presence of significant breathlessness. If successful, the patient was discharged from the ED, otherwise chest tube drainage (CTD) was employed.

Among patients with SSP, most were treated with CTD, but small pneumothoraces in the absence of breathlessness were managed conservatively. Those requiring CTD had a Rocket chest drain (size 12-French) inserted at the bedside in the axillary safe triangle via aseptic Seldinger technique, with the aid of local anaesthesia. The chest tube was attached to an underwater seal bottle and the patient referred to the respiratory team. Following review and confirmation of suitability for the ambulatory pathway, the chest tube was disconnected from the underwater seal and attached to a Heimlich type one-way flutter valve (Pneumostat, Atrium Medical, Hudson, New Hampshire, USA).

Patients were shown how to check for an air leak by squirting water into the air leak well. They were instructed to perform this daily if possible, and shown how to drain the Pneumostat fluid chamber if it filled with pleural fluid. They were allowed home after a chest radiograph with a patient information leaflet including clear instructions on who to contact should any problems arise. Patients were advised to take simple analgesia like paracetamol and/or ibuprofen regularly, and those with more severe pain were prescribed co-codamol 30/500 and/ or morphine sulfate (oramorph) $10 \mathrm{mg} / 5 \mathrm{~mL}$ liquid as required. All patients were then reassessed on alternate days on the ambulatory care unit (including weekends), with a chest radiograph on arrival. Patients were actively dissuaded from visiting their general practitioners (GPs) for any drain-related issues, and instead asked to contact the ambulatory care team directly. Unexpected visits to the ED and GPs were monitored.

Once the air leak had resolved for 24 hours and chest radiography confirmed lung re-expansion, the chest tube was removed and the patient discharged from follow-up. Those with an ongoing air leak on day 5 were referred to the local thoracic surgery unit in Leicester (Glenfield Hospital) and admitted electively from home for surgery with their chest drain in situ. Suction was not employed in any patient.

On completion of treatment, patients were asked to indicate their average pain during ambulatory management on a numeric intensity scale $(0-10)$. Their satisfaction scores were also recorded, on a $1-5$ scale, with 5 being the most satisfied.

\section{Primary outcome}

The primary outcome was SSP resolution by day 5, defined as complete re-expansion of the lung on chest radiograph with no air leak. This was compared with the resolution rate in PSP (comparator group).

\section{Secondary outcomes}

1. Number of days spent with chest drain in situ.

2. Persistent air leak requiring surgery.

3. Complications including drain dislodgement, blockage and empyema.

4. Pain and satisfaction scores during ambulatory management.

5. Early recurrence $(<1$ week $)$.

6. Cost savings.

As the ambulatory management of PSP is widely accepted as being safe, we used this group as a comparator group to assess the outcomes of patients with SSP. For 


\begin{tabular}{|c|c|c|c|}
\hline & All & PSP & SSP \\
\hline $\begin{array}{l}\text { Total number of } \\
\text { patients }\end{array}$ & 163 & 64 & 99 \\
\hline $\begin{array}{l}\text { Number treated } \\
\text { on ambulatory } \\
\text { pathway }\end{array}$ & 111 & 62 & 49 \\
\hline $\begin{array}{l}\text { Chest tube } \\
\text { drainage (\%) }\end{array}$ & $73(66)$ & $29(47)$ & $44(90)$ \\
\hline $\begin{array}{l}\text { Age, year, median } \\
\text { (range) }\end{array}$ & $39(16-76)$ & $31(16-47)$ & $65(21-76)$ \\
\hline Male, n (\%) & $70(63)$ & $44(71)$ & $26(53.1)$ \\
\hline $\begin{array}{l}\text { Pneumothorax } \\
\text { side: right/left }\end{array}$ & $61 / 50$ & $34 / 28$ & $27 / 22$ \\
\hline $\begin{array}{l}\text { Pneumothorax } \\
\text { size large/small }\end{array}$ & $81 / 30$ & $42 / 20$ & $40 / 9$ \\
\hline First episode (\%) & $85(77)$ & 49 (79) & $36(73)$ \\
\hline Recurrences (\%) & $26(23)$ & $13(21)$ & $13(27)$ \\
\hline
\end{tabular}

PSP, primary spontaneous pneumothorax; SSP, secondary spontaneous pneumothorax.

statistical comparison, t-tests and Mann-Whitney U tests were used for continuous data depending on whether they were normally distributed, and the $\chi^{2}$ test for binary outcomes. For all tests, a significance threshold of 0.05 was applied.

\section{RESULTS}

\section{Patient characteristics}

Table 1 outlines the patient characteristics. During the audit period, of the 163 consecutive patient episodes evaluated (figure 1), 99 were SSP and 64 were PSP. Of the 99 SSP, 49 patients received outpatient management. The remainder, who were ineligible due to failure to meet the predefined inclusion/exclusion criteria, received usual inpatient care. This also included six patients who were eligible but whose lungs had re-expanded prior to involvement of the respiratory team. As their drains were removed prior to discharge, they were not included in the ambulatory cohort. Among the patients with SSP managed by the ambulatory service, 36 (73\%) were first episodes and the remaining $13(27 \%)$ were recurrences. Forty $(82 \%)$ had large pneumothoraces. The median age was 65 (range 21-76). Forty-four (90\%) patients were treated with CTD, and the other five who had small asymptomatic pneumothoraces were observed as outpatients.

For the 64 patients with PSP, all but 2 were treated on the ambulatory pathway. Of those who were admitted, one was pregnant and reluctant to go home with a drain, and the other had a hydropneumothorax. The 62 (97\%) patients with PSP treated on the ambulatory pathway had a median age of 31 years (range 16-47) and $71 \%$ were male. Forty-two patients $(68 \%)$ had a large PSP. Twenty-nine patients (47\%) required CTD, and the remaining $33(53 \%)$ were either successfully aspirated or treated conservatively.

\section{Primary outcome}

The primary outcome of complete radiographic resolution with no air leak by day 5 was similar in both groups. Sixty-five per cent (32 of the 49) in the SSP had complete resolution by day 5 vs $79 \%$ (49 of the 62 ) in the PSP group ( $\mathrm{p}=0.108)$.

\section{Secondary outcomes}

The mean drainage time with a one-way valve in situ in patients with SSP was 5.84 days. In comparison, the PSP

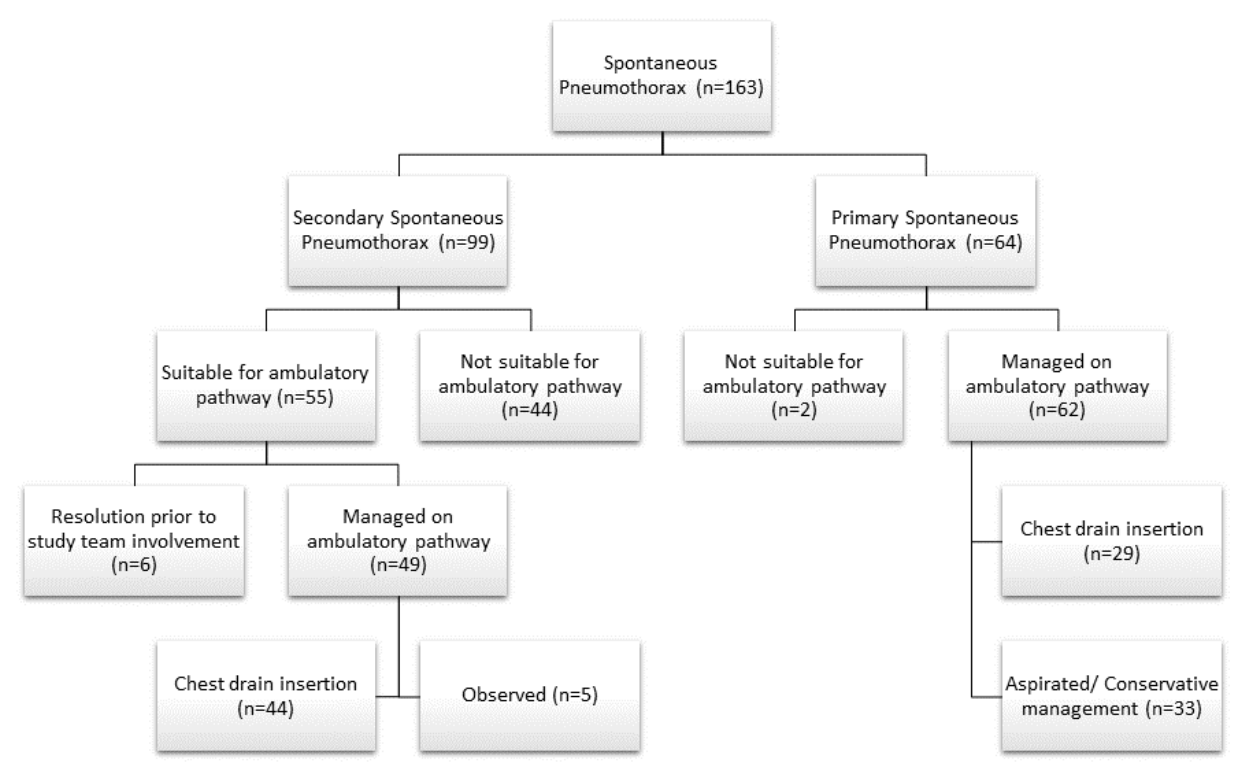

Figure 1 Study flow diagram. 
group had a mean of 5.69 days, representing a difference of 0.15 days $(95 \%$ CI -2.47 to 2.16 ; $\mathrm{p}=0.897)$.

Among the 17 patients with SSP with ongoing air leak on day 5, 9 were referred to the thoracic surgeons for further management. The remaining eight declined referral for surgery and healed spontaneously. The median duration of drainage in these eight patients was 8 days (range 7-25 days). Most patients who refused surgery had pleurodesis with talc slurry after being readmitted electively for an overnight stay. There were 13 patients in the PSP group who had non-resolution on day 5. Of these, 11 were referred to the thoracic surgeons for further management. The other two patients had cessation of air leak with complete lung re-expansion by days 6 and 8, respectively. They were unwilling to be referred for surgery.

Complications were scarce in both groups. There were five drain blockages, all requiring drain replacement: three episodes occurred in patients with SSP and two episodes in the PSP group. All these patients with drain blockage were asymptomatic, and the complication was identified during routine review.

One drain was displaced inadvertently while a patient with SSP attempted to empty pleural fluid from the Pneumostat chamber. As his lung had already re-expanded, the drain did not require replacement. Two patients developed empyema; one of these was in a patient who had declined surgery and required prolonged drainage (19 days). His air leak healed soon after he developed the infection and the drain was eventually removed on day 25. Another patient developed empyema (purulent effusion) but remained asymptomatic throughout and was managed with oral flucloxacillin.

The mean pain scores (scale $0-10$ ) were 3.65 in the SSP group and 3.78 in the PSP group, a difference of -0.13 (95\% CI -1.15 to $1.4 ; \mathrm{p}=0.842$ ). Thirty-two per cent of SSP and $41 \%$ of PSP required stronger prescribed analgesia (co-codamol or oramorph). The satisfaction scores (scale 0-5) were excellent, with a mean score of 4.95 in the SSP and 4.79 in the PSP, representing a difference of 0.16 (95\% CI -0.33 to $0.13 ; \mathrm{p}=0.07)$.

Patients in the SSP group had a mean of 2.16 visits to the ambulatory care unit during their management, compared with 2.07 attendances in the PSP group. There were a total of seven unplanned visits in the SSP group. Most of these were for simple issues such as dressing change, pain or to collect syringes to drain the Pneumostat device. Another patient attended with minor haemoptysis and had a CT demonstrating minor collapse/consolidation. She did not require admission. There were two unplanned reviews in patients with PSP due to pain.

Of the 36 patients with a first episode of SSP, 5 (14\%) had an ipsilateral recurrence within the first 12 months, as opposed to 6 out of $49(12 \%)$ patients with a first episode of PSP. In total, there were just three (2.7\%) early recurrences within 1 week following drain removal, two of which were in the SSP cohort (table 2).

\begin{tabular}{|c|c|c|c|c|}
\hline & All & PSP & SSP & $\begin{array}{l}P \\
\text { value }\end{array}$ \\
\hline $\begin{array}{l}\text { Resolution by } \\
\text { day } 5(\%)\end{array}$ & $81(73)$ & $49(79)$ & $32(65)$ & 0.108 \\
\hline $\begin{array}{l}\text { Total duration } \\
\text { of drainage, } \\
\text { days }\end{array}$ & $5.78 \pm 0.56$ & $5.69 \pm 0.82$ & $5.84 \pm 0.77$ & 0.897 \\
\hline $\begin{array}{l}\text { Inpatient } \\
\text { hospital stay, } \\
\text { days }\end{array}$ & $0.44 \pm 0.09$ & $0.18 \pm 0.05$ & $0.74 \pm 0.17$ & 0.001 \\
\hline $\begin{array}{l}\text { Number of } \\
\text { complications }\end{array}$ & 8 & 2 & 6 & 0.089 \\
\hline Pain score & $3.7 \pm 0.31$ & $3.78 \pm 0.52$ & $3.65 \pm 0.39$ & 0.842 \\
\hline $\begin{array}{l}\text { Satisfaction } \\
\text { score }\end{array}$ & $4.89 \pm 0.04$ & $4.79 \pm 0.1$ & $4.95 \pm 0.04$ & 0.069 \\
\hline $\begin{array}{l}\text { Recurrence } \\
\text { within } 1 \text { week } \\
\text { (\%) }\end{array}$ & $3(2.7)$ & $1(1.6)$ & $2(4)$ & 0.452 \\
\hline
\end{tabular}

PSP, primary spontaneous pneumothorax; SSP, secondary spontaneous pneumothorax.

\section{Healthcare cost estimates}

For patients on the ambulatory pathway with a chest tube and Pneumostat device, who would have otherwise required an inpatient stay, we calculated cost savings. Patients with PSP who were discharged after being treated conservatively with either observation or successful aspiration were excluded from this analysis. During the study period of just under 3 years, we estimated savings of $£ 86$ 796 , equating to $£ 1188.80$ per patient. Table 4 details the breakdown.

\begin{tabular}{|c|c|c|c|}
\hline $\begin{array}{l}\text { Cost/Savings } \\
\text { of ambulatory } \\
\text { management }\end{array}$ & $\mathbf{n}$ & $\begin{array}{l}\text { Unit cost } \\
\text { (£) }\end{array}$ & $\begin{array}{l}\text { Total savings/ } \\
\text { costs for } \\
\text { ambulatory } \\
\text { management (£) }\end{array}$ \\
\hline Bed days saved & 379 & 329 & 121280 \\
\hline $\begin{array}{l}\text { Cost of } \\
\text { attendances to } \\
\text { ambulatory care }\end{array}$ & 160 & 200 & 32000 \\
\hline $\begin{array}{l}\text { Cost of } \\
\text { attendances } \\
\text { to emergency } \\
\text { department }\end{array}$ & 1 & 160 & 160 \\
\hline $\begin{array}{l}\text { Cost of } \\
\text { Pneumostat } \\
\text { devices }\end{array}$ & 83 & 28 & 2324 \\
\hline Overall savings & & & $\begin{array}{l}£ 86796(\$ 113 \\
920) \\
£ 1188.80 \text { per } \\
\text { patient }(\$ 1550)\end{array}$ \\
\hline
\end{tabular}




\section{DISCUSSION}

This study suggests that the use of chest drains with one-way valves in the ambulatory management of carefully selected SSP may be safe and cost-saving and appears to be as effective as the ambulatory management of PSP.

Among the patients with ambulatory management of SSP, 32 of the $49(65 \%)$ resolved by day 5 . There are no published figures for inpatient management of this specific group of patients with SSP. For those with PSP, 49 of the $62(79 \%)$ had resolution by day 5 . This is in keeping with published figures of $80 \%$ for inpatient management. ${ }^{9}$

The mean total drainage time in the ambulatory SSP group was 5.84 days, which was statistically similar to a mean total drainage time of 5.69 days in the ambulatory PSP group. This compares well with a mean drainage of 8 days for inpatient management of PSP. ${ }^{13}$ Of the total drainage time, a mean of 0.74 days in the SSP group and 0.18 days in the PSP group were spent as inpatient.

Safety concerns are likely to be the main barrier to the uptake of outpatient pneumothorax management. Our experience however refutes this. There were no serious incidents throughout the 3-year audit period. Only eight complications $(10.25 \%)$ were encountered in total. This compares well with published rates of up to $30 \%{ }^{13}$ for patients managed in hospital. Five patients had blocked drains which were replaced without any adverse outcome. It is important patients are educated about their devices and know how and when to seek attention. There was one episode of a chest drain inadvertently being removed. Again, this compares favourably with published figures of at least $20 \%$ of chest drains being inappropriately displaced. ${ }^{13}$ This advantage is likely to be related to the lightweight Pneumostat device, which replaces the lengthy tubing and heavy drain bottle conventionally used as an underwater seal.

In our study, a respiratory physician discharged patients shortly after chest drain insertion and review. No patients in our study presented with or developed tension pneumothorax. Unlike some other studies, we did not use thoracic suction. Our previous experience suggests that the vast majority of deflated lungs re-expand with time without suction when low resistance devices such as Heimlich valves are used. Our resolution rates on day 5 appear to confirm this.

In patients with a first episode of SSP, $14 \%$ had an ipsilateral recurrence within the first 12 months, as opposed to $12 \%$ in patients with a first episode of PSP. This low recurrence rate compares favourably with 1-year recurrence rate of $17 \%{ }^{9}$ quoted in the literature.

While direct comparison between ambulatory and non-ambulatory SSP patients is not possible due to severe comorbidities and respiratory failure in most of the patients in the non-ambulatory group, data for the whole cohort of patients with SSP have been presented for information only (table 3 ). To the best of our knowledge, there are no data in the published literature on outcomes in patients with SSP.
Table 3 Comparison of ambulatory SSP vs nonambulatory SSP

Ambulatory
SSP

$\begin{array}{lll}\text { Total number of patients } 49 & 50 \\ \text { Chest tube drainage } \quad 44(90) & 47(94)\end{array}$

(\%)

\begin{tabular}{lll}
$\begin{array}{l}\text { Age, year, median } \\
\text { (range) }\end{array}$ & $65(21-76)$ & $74.5(30-92)$ \\
\hline Male, $\mathrm{n}(\%)$ & $26(53.1)$ & $38(76)$ \\
\hline $\begin{array}{l}\text { Pneumothorax size: } \\
\text { large/small }\end{array}$ & $27 / 22$ & $32 / 18$ \\
\hline $\begin{array}{l}\text { Resolution by day } 5(\%) \\
\text { Duration of drainage, } \\
\text { days }\end{array}$ & $5.84 \pm 0.77$ & $32(64)$ \\
\hline
\end{tabular}

SSP, secondary spontaneous pneumothorax.

Our protocol was less expensive than traditional CTD management with hospitalisation. We have previously shown that outpatient management is cost-saving. ${ }^{14}$ During the audit period alone, we estimated savings of around $£ 86796$, equating to a crude estimate of around $£ 1188$ per patient. In addition to the direct savings, other advantages of our approach include reduction in hospital stay with its associated complications, and indirect cost savings such as days off from work (some continued working from home) and childcare costs. Questionnaire (supplementary material 1) responses from patients confirm a positive experience. Even though indirect costs to the patient such as multiple trips to the hospital, taxis and parking were not calculated, these would be offset by the same costs being incurred by the patient's family during their visits to the hospital.

There are limitations to this study, however. It is a non-randomised, single-centre experience, with lack of a formal 'standard care' control group. Published data for comparison are also limited. As a result, we used the ambulatory PSP cohort as a comparator group despite itself being a novel approach with limited published data. In addition, only 49 of the 99 patients with SSP could be managed on the ambulatory pathway unlike nearly all patients with PSP. This is due to the wide variability in the health status of patients with SSP, and emphasises the importance of careful patient selection for management on an ambulatory pathway.

There is a growing consensus that selected patients with PSP can be managed safely in an outpatient setting. This study suggests that selected patients with SSP do equally well, without additional complications. To the best of our knowledge, this is the largest data set describing prospectively the ambulatory management of SSP. High-quality randomised controlled trials are required to confirm the findings.

Contributors All authors contributed to the authorship of the document. FK wrote the paper and did all the statistical analyses. RR collected all the data. RR, YV and 
MN designed and conducted the study, as well as revised the paper. All authors approved the final work.

Funding The authors have not declared a specific grant for this research from any funding agency in the public, commercial or not-for-profit sectors.

Competing interests None declared.

Patient consent Not required.

Ethics approval As this was an evaluation of our service, the institution's R\&D office had confirmed that research approval was not required.

Provenance and peer review Not commissioned; externally peer reviewed.

Data sharing statement No additional data are available.

Open access This is an open access article distributed in accordance with the Creative Commons Attribution Non Commercial (CC BY-NC 4.0) license, which permits others to distribute, remix, adapt, build upon this work non-commercially, and license their derivative works on different terms, provided the original work is properly cited, appropriate credit is given, any changes made indicated, and the use is non-commercial. See: http://creativecommons.org/licenses/by-nc/4.0/.

\section{REFERENCES}

1. Melton LJ, Hepper NG, Offord KP. Incidence of spontaneous pneumothorax in Olmsted County, Minnesota: 1950 to 1974. Am Rev Respir Dis 1979;120:1379-82.

2. Gupta D, Hansell A, Nichols T, et al. Epidemiology of pneumothorax in England. Thorax 2000;55:666-71.

3. MacDuff A, Arnold A, Harvey J, et al. Management of spontaneous pneumothorax: British Thoracic Society pleural disease guideline 2010. Thorax 2010;65(Suppl 2):ii18-31.
4. Baumann MH, Strange C, Heffner JE, et al. Management of spontaneous pneumothorax: an American College of chest physicians Delphi consensus statement. Chest 2001;119:590-602.

5. De Leyn P, Lismonde M, Ninane V, et al. Guidelines Belgian Society of Pneumology. Guidelines on the management of spontaneous pneumothorax. Acta Chir Belg 2005;105:265-7.

6. Bernstein A, Waqaruddin M, Shah M. Management of spontaneous pneumothorax using a Heimlich flutter valve. Thorax 1973;28:386-9.

7. Mercier C, Page A, Verdant A, et al. Outpatient management of intercostal tube drainage in spontaneous pneumothorax. Ann Thorac Surg 1976;22:163-5.

8. Marquette $\mathrm{C}-\mathrm{H}$, Marx A, Leroy S, et al. Simplified stepwise management of primary spontaneous pneumothorax: a pilot study. Eur Respir J 2006;27:470-6.

9. Massongo M, Leroy S, Scherpereel A, et al. Outpatient management of primary spontaneous pneumothorax: a prospective study. Eur Respir J 2014;43:582-90.

10. Voisin F, Sohier L, Rochas $\mathrm{Y}$, et al. Ambulatory management of large spontaneous pneumothorax with pigtail catheters. Ann Emerg Med 2014;64:222-8.

11. Brims FJH, Maskell NA. Ambulatory treatment in the management of pneumothorax: a systematic review of the literature. Thorax 2013;68:664-9.

12. Oken MM, Creech RH, Tormey DC, et al. Toxicity and response criteria of the Eastern Cooperative Oncology Group. Am J Clin Oncol 1982;5:649-56.

13. Davies HE, Merchant S, McGown A. A study of the complications of small bore 'Seldinger' intercostal chest drains. Respirology 2008;13:603-7.

14. Pillay C, Shah B, Naeem M, et al. Cost effectiveness of ambulatory management of spontaneous pneumothorax. Thorax 2015;70(Suppl 3):A165-6. 